\title{
A Systematic Review and Meta-Analysis of Arthroscopic Meniscus Repair in Young Patients: Comparison of All- Inside and Inside-Out Suture Techniques
}

\author{
Dong-Geun Kang, $\mathrm{MD}^{1}$, Young-Jin Park, $\mathrm{MD}^{2}$, Jae-Ha Yu, $\mathrm{MD}^{3}$, Jong-Byung $\mathrm{Oh}, \mathrm{MD}^{3}$, and Dong-Yeong Lee, $\mathrm{MD}^{3}$ \\ ${ }^{1}$ Department of Orthopaedic Surgery, Gyeongsang National University Changwon Hospital, Gyeongsang National University School of Medicine, Changwon; \\ ${ }^{2}$ Department of Orthopaedic Surgery, Gyeongsang National University Hospital, Gyeongsang National University School of Medicine, Jinju; ${ }^{3}$ Department of \\ Orthopaedic Surgery, The Armed Forces Daegu Hospital, Gyeongsan, Korea
}

\begin{abstract}
Purpose: The purposes of the present study were to review published studies that investigated arthroscopic meniscus repair to treat meniscus injury in young patients and to compare all-inside and inside-out suture techniques.

Methods: Various electronic databases were queried for published articles, and this search was updated in August 2017 for evaluating the outcomes of arthroscopic meniscus surgery in young patients. Data search, extraction, analysis, and quality assessment were performed according to the Cochrane Collaboration guidelines, and the clinical outcomes were evaluated using various outcome values in young patients according to suture techniques. Results: Three randomized controlled trials and three prospective comparative studies were included in this systematic review and meta-analysis. There were no significant differences in clinical outcomes such as meniscus healing rate (risk ratio [RR], 1.11; $95 \%$ confidence interval [CI], 0.90 to 1.37 ; $\mathrm{I}^{2}=39 \%$ ) and perioperative complications (RR, $0.62 ; 95 \%$ CI, 0.23 to $1.72 ; \mathrm{I}^{2}=43 \%$ ) between all-inside and inside-out techniques for meniscus repair. Conclusions: The present study shows favorable results for clinical outcomes such as meniscus healing rate and perioperative complications in young patients. Furthermore, based on our results, both all-inside and inside-out meniscal suture techniques are equally effective in these patients.
\end{abstract}

Keywords: Knee, Meniscus, Tear, Repair, Suture technique

\section{Introduction}

A meniscus tear in young, active patients is critical because of its clinical consequences. The meniscus acts as a shock-absorbing, load-transmitting, and secondary anterior stabilizer of the knee, plays a role in proprioception, and contributes to the lubrication

Received October 13, 2017; Revised January 15, 2018;

Accepted February 19, 2018

Correspondence to: Dong-Yeong Lee, MD

Department of Orthopaedic Surgery, The Armed Forces Daegu Hospital,

Daegyeong-ro 425-41, Hayang-eup, Gyeongsan 38427, Korea

Tel: +82-53-750-1777, Fax: +82-53-854-0822

E-mail: whatttary@hanmail.net

Source of funding: This work was supported by the Korean Military Medical Research Project funded by the ROK Ministry of National Defense (ROK-MND-2017-KMMRP-025).

This is an Open Access article distributed under the terms of the Creative Commons Attribution Non-Commercial License (http://creativecommons.org/licenses/by-nc/4.0/) which permits unrestricted non-commercial use, distribution, and reproduction in any medium, provided the original work is properly cited. and nutrition of the articular cartilage ${ }^{1-5)}$. For these reasons, a meniscal injury is a potential risk factor for knee osteoarthritis, and thus, many orthopedic surgeons advocate performing surgical meniscal procedures to treat these lesions.

Currently, the typical meniscus surgery is arthroscopicallyassisted meniscectomy or meniscus repair. Compared with open meniscus surgery, arthroscopic meniscus surgery has various beneficial effects such as short operation time, early recovery, and minimal trauma. Despite these benefits, arthroscopic meniscectomy can cause disruption of the circumferential fibers, which can ultimately lead to the inability of the remaining meniscus to effectively control hoop stress ${ }^{6}$. In addition, removing meniscal tissue can directly increase contact stresses, which can cause degenerative knee disorders such as osteoarthritis ${ }^{7-9)}$. Based on this theoretical evidence, a recent review ${ }^{10)}$ suggested that meniscal repair has better long-term patient-reported outcomes and better activity levels than meniscectomy; thus, arthroscopic meniscus repair is recommended over meniscectomy in young patients.

Several recently developed meniscal suture devices are avail- 


\section{Kang et al. All-Inside vs. Inside-Out Suture in Arthroscopic Meniscus Repair}

able for peripheral meniscus tears ${ }^{11-15)}$. The development of these devices decreased the risk of meniscectomy, which led to less secondary osteoarthritis ${ }^{16-18)}$. In particular, the all-inside technique has advantages such as low risk of neurovascular injury and short operation time ${ }^{19)}$. However, despite these benefits, recent randomized controlled trials $(\mathrm{RCTs})^{20)}$ reported that there were no statistically significant differences in measured outcomes between the all-inside and inside-out suture techniques, whereas other RCTs ${ }^{21)}$ suggested that arthroscopic meniscus repair with the inside-out technique was superior in comparison with the other methods. Many controversies remain, and therefore, the purposes of the present study were (1) to evaluate the effectiveness of arthroscopic meniscus repair in young patients and (2) to compare the clinical outcomes between the all-inside and inside-out suture techniques. We hypothesized that young patients treated with arthroscopic meniscus repair would also have favorable outcomes and that the two suture techniques were not significantly different in clinical outcomes.

\section{Methods}

\section{Study Selection}

To identify the relevant studies, we used the controlled vocabulary and free text provided in Appendix 1 to query MEDLINE, EMBASE, the Cochrane Central Register of Controlled Trials. This study is based on the Cochrane Review Methods, and reporting was carried out according to Preferred Reporting Items for Systematic Reviews and Meta-Analyses (PRISMA) guidelines. We attempted to identify all relevant studies in the English language literature irrespective of the publication type (articles, posters, conference articles, instructional course lectures, etc.), publication journal, and publication date. We updated this search in August 2017, and it now includes reference lists from the studies and any review articles that we identified. The reference lists of the investigated studies were scrutinized to identify any possible additional publications not found through electronic or manual searches. Since unpublished data have the risk of bias, they were not included in this study.

\section{Eligibility Criteria}

We included studies in our meta-analysis if (1) the subjects were patients who had received arthroscopic meniscus repair in young age (the subjects' mean age in included studies was less than 30 years), (2) the studies investigated clinical outcomes after arthroscopic meniscus repair in young patients, (3) the studies reported on a minimum two-year follow-up data on clinical outcome, functional and imaging outcomes, and (4) the studies included only level I or II evidence. However, we excluded subjects who had degenerative meniscus lesions, studies that only described surgical techniques, studies regarding revision surgery, subjects who had congenital disease or congenital deformity such as discoid meniscus lesions, studies that reported on less than two years of follow-up data, intraoperative measures, or nonclinical outcome measures, levels III, IV, or V, and in vitro and animal studies. Detailed criteria are presented in Table 1.

\section{Data Collection and Analysis}

We independently assessed the titles or abstracts of the studies identified via the query and then assessed the full papers for final inclusion through discussion and consensus. We independently abstracted the eligible data into predefined formats and checked them for accuracy. We collected information on the study characteristics (information about the authors, journal, country, surgical procedure, study design, level of evidence, publication year, sample size, subjects' age, sex, and follow-up period) (Table 2). We evaluated the clinical results of the included studies with

Table 1. Inclusion and Exclusion Criteria

\begin{tabular}{|c|c|}
\hline Inclusion criteria & Exclusion criteria \\
\hline $\begin{array}{l}\text { The subjects were young patients that received arthroscopic meniscus } \\
\text { repair (the subjects' mean age in included studies was less than } 30 \\
\text { years) }\end{array}$ & $\begin{array}{l}\text { Studies that did not compare the effect of arthroscopic meniscus repair } \\
\text { Studies regarding revision surgery } \\
\text { Subjects who had congenital disease or congenital deformity }\end{array}$ \\
\hline $\begin{array}{l}\text { The studies evaluated clinical outcomes after arthroscopic meniscus } \\
\text { repair in young patients }\end{array}$ & $\begin{array}{l}\text { Studies reporting less than 2-year follow-up data on clinical outcome, } \\
\text { functional and imaging outcomes }\end{array}$ \\
\hline $\begin{array}{l}\text { Studies reporting a minimum 2-year follow-up data on clinical outcome, } \\
\text { functional and imaging outcomes }\end{array}$ & $\begin{array}{l}\text { Level III, IV and V evidence (case report, technical note, letters to } \\
\text { editor), review articles }\end{array}$ \\
\hline Only included level-I or -II evidence & Studies that only reported non-clinical outcome measures or \\
\hline No exclusions were made on the basis of language & intraoperative measures were excluded \\
\hline Human subjects & Animal studies or in vitro studies \\
\hline
\end{tabular}


Table 2. Characteristics of the Included Studies

\begin{tabular}{|c|c|c|c|c|c|c|c|c|c|c|}
\hline Study & Journal & Country & $\begin{array}{l}\text { Surgical } \\
\text { procedure }\end{array}$ & $\begin{array}{l}\text { Study } \\
\text { design }\end{array}$ & $\begin{array}{l}\text { Level of } \\
\text { evidence }\end{array}$ & Year & $\begin{array}{l}\text { Sample } \\
\text { size }\end{array}$ & $\begin{array}{l}\text { Age } \\
(\mathrm{yr})\end{array}$ & $\begin{array}{l}\text { Sex } \\
(\mathrm{M}: \mathrm{F})\end{array}$ & $\begin{array}{l}\text { Follow-up } \\
\text { time (mo) }\end{array}$ \\
\hline $\begin{array}{l}\text { Albrecht-Olsen } \\
\text { and Bak }\end{array}$ & $\begin{array}{l}\text { Knee Surgery Sports } \\
\text { Traumatology } \\
\text { Arthroscopy }\end{array}$ & Denmark & MR & RCT & I & 1999 & 68 & $26.0(18-40)$ & $55: 13$ & Not provided \\
\hline Spindler et al. ${ }^{24)}$ & $\begin{array}{l}\text { American Journal of } \\
\text { Sports Medicine }\end{array}$ & USA & MR & PCS & II & 2003 & 125 & $23.8 \pm 9.3$ & $65: 60$ & 40.1 \\
\hline Barber et al. ${ }^{26)}$ & Arthroscopy & USA & MR & PCS & II & 2005 & 85 & $27.0(15-48)$ & $54: 31$ & $26.5(12-56)$ \\
\hline Hantes et al. ${ }^{21)}$ & $\begin{array}{l}\text { Knee Surgery Sports } \\
\text { Traumatology } \\
\text { Arthroscopy }\end{array}$ & Greece & MR & RCT & II & 2006 & 57 & 27.1 & $13: 44$ & $22.3(17-37)$ \\
\hline Bryant et al. ${ }^{20)}$ & $\begin{array}{l}\text { American Journal of } \\
\text { Sports Medicine }\end{array}$ & Canada & MR & RCT & I & 2007 & 100 & $25.4 \pm 8.0$ & $62: 38$ & $28.0 \pm 8.4(9-46)$ \\
\hline Choi et al. ${ }^{27)}$ & $\begin{array}{l}\text { American Journal of } \\
\text { Sports Medicine }\end{array}$ & Korea & MR & PCS & II & 2009 & 48 & $28.0(15-55)$ & $44: 4$ & 35.7 (24-91) \\
\hline
\end{tabular}

Values are presented as mean \pm standard deviation (range).

MR: meniscus repair, RCT: randomized controlled trial, PCS: prospective cohort study, M: male, F: female.

respect to the operated side, suture technique, tear length, tear zone, concomitant anterior cruciate ligament (ACL) reconstruction, time from injury to repair, complications, healing rate, and outcome scales at final follow-up (Table 3). We also investigated the numbers of subjects or means and standard deviations for the demographic data and clinical outcomes between the groups.

\section{Assessing Methodological Quality}

We independently assessed the methodological qualities of the RCTs using the PEDro critical appraisal scoring system, which is an 11-item scale that has previously demonstrated reliability and validity in assessing RCTs ${ }^{22)}$. In addition, we assessed the methodological quality of comparative studies using the Newcastle-Ottawa quality assessment scale ${ }^{23)}$. We resolved any disagreements between us through discussion or review by a third investigator, and we attempted to clarify any uncertainties in outcomes or methodologies through personal correspondence with the authors. We did not evaluate publication bias due to low statistical power because the number of studies included was less than 10 in each field of research.

\section{Statistical Analysis}

In this review, we evaluated the clinical outcomes of arthroscopic meniscus surgery in young patients and investigated the effects of different suture techniques; clinical outcomes mainly focused on meniscus healing rate and perioperative complications. To evaluate the two outcomes after arthroscopic meniscus surgery, we calculated the risk ratios (RRs) between the groups. We used
Review Manager (RevMan) ver. 5.3 (The Cochrane Collaboration, London, UK) to estimate the overall pooled effect sizes for each outcome and conducted a meta-analysis of the included studies using a random effects model. For binary outcomes, we calculated the RRs between the groups using the MantelHaenszel method. We assessed statistical heterogeneity among the studies according to the I-squared $\left(\mathrm{I}^{2}\right)$ value, with values of $25 \%, 50 \%$, and $75 \%$ considered low, moderate, and high, respectively. We used the Cochrane Q statistic (chi-square test) to assess heterogeneity and defined $\mathrm{p}<0.10$ as significant heterogeneity.

\section{Results}

\section{Identifying the Studies}

We initially identified a total of 3,201 relevant articles; of these, 177 were duplicated in the databases. After we screened the remaining 3,024 articles using titles and abstracts, we excluded all but 13 because they were not relevant to the purpose of the present study. A thorough full-text review of the 13 articles excluded 7 because they lacked vital data such as experimental or clinical outcomes. The majority of the excluded articles did not investigate the clinical outcomes of each technique, were inappropriate regarding the patients' ages, evaluated meniscus suture techniques, were biomechanical studies of meniscus, or were animal studies. Finally, we included six studies for the data extraction

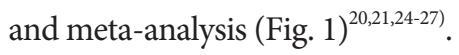




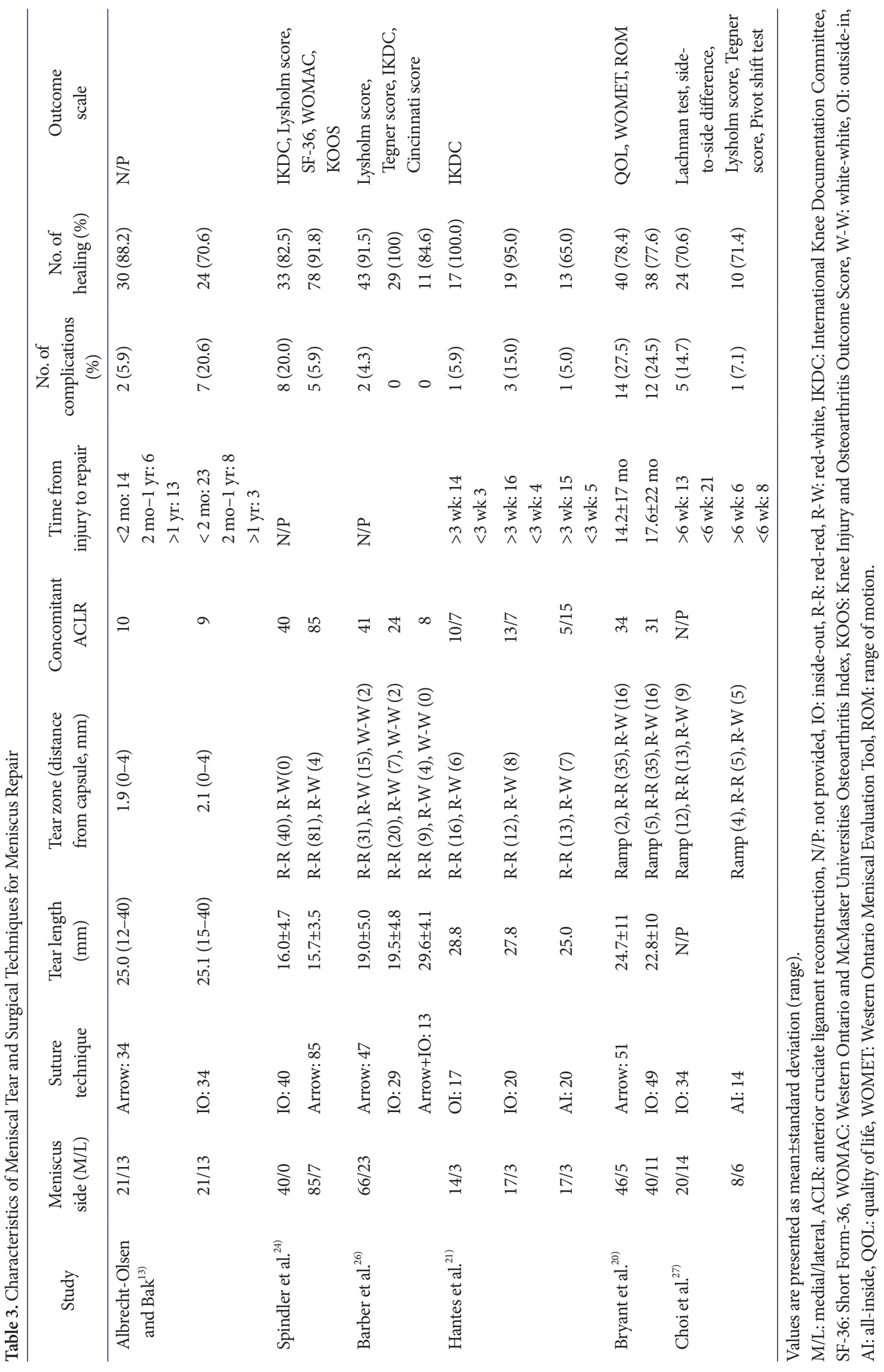




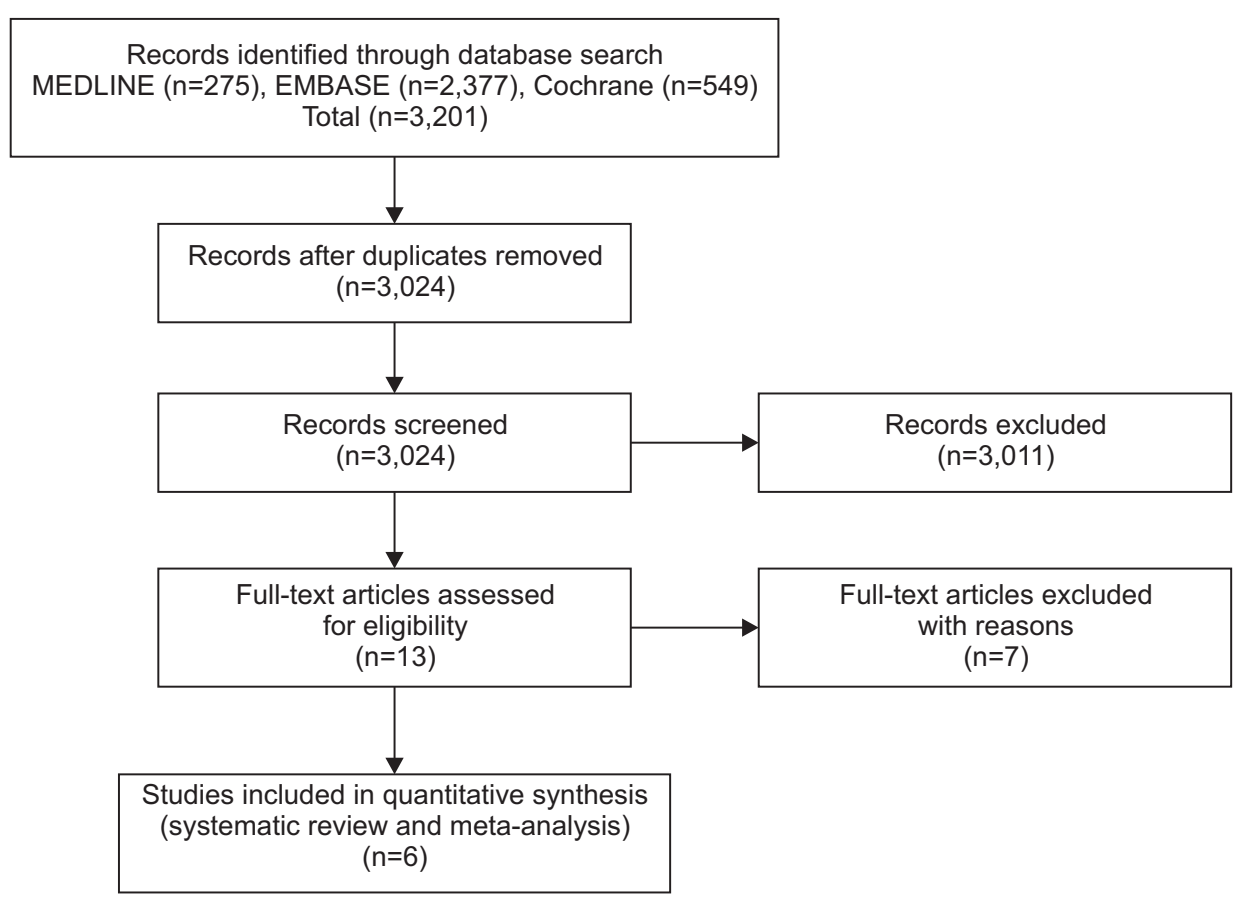

Fig. 1. Preferred Reporting Items for Systematic Reviews and Meta-Analyses (PRISMA) flow diagram.

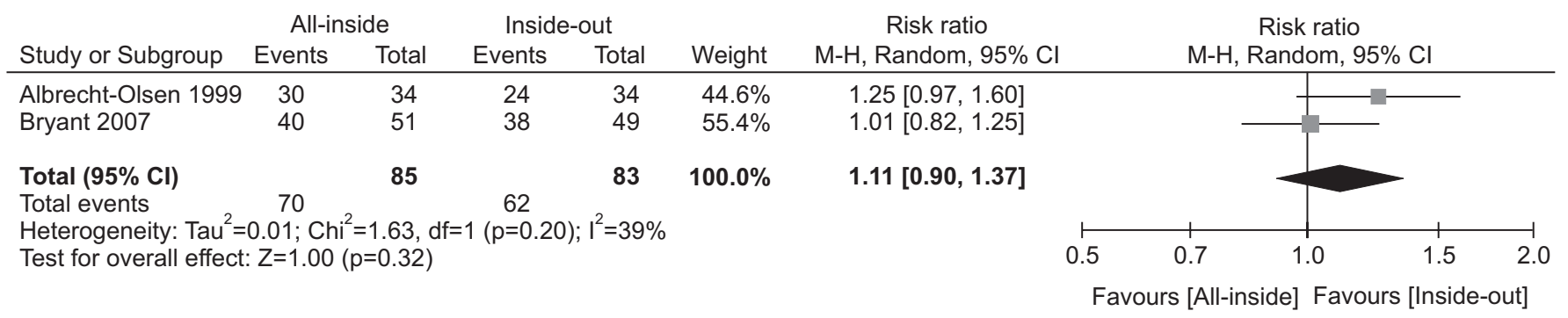

Fig. 2. Forest plot of meniscus healing rates of randomized controlled trials using the all-inside and inside-out techniques. CI: confidence interval.

\section{Quality of the Included Studies}

As mentioned above, we used the PEDro critical appraisal score to assess the methodological quality of the RCTs and assessed the methodological quality of comparative studies using the Newcastle-Ottawa Quality Assessment Scale. The mean PEDro score was 8.3 points (range, 8 to 9 points), indicating that most studies had good quality by the current scoring system. Furthermore, total scores of the Newcastle-Ottawa quality assessment scale are over 7.3 points (range, 7 to 8 points), which indicated a low risk of bias for the included studies, and therefore, we included all selected studies in this meta-analysis.

\section{Clinical Results of the RCTs}

The six studies were three RCTs and three prospective comparative studies. It is inappropriate to analyze RCTs with comparative studies because this increases the risk of bias, and thus, we only analyzed RCTs. We could not analyze outcome scales such as Lysholm knee score, Tegner activity score, International Knee Documentation Committee score, quality of life, or side-to-side differences because of insufficient data such as means or standard deviations. Based on tables and results of each study, we prepared a forest plot of healing rate and prevalence of perioperative complications.

\section{1) Healing rate}

In qualitative analysis, regardless of suture technique, the healing rates for arthroscopic meniscus repair were 70.8\%-93.3\% across the studies. In the quantitative analysis, the two RCTs ${ }^{20,25)}$ reported on the healing rates between the two techniques with a total of 168 patients (85 in the all-inside group and 83 in the inside-out group). There was no significant difference in the healing rate between the all-inside and inside-out techniques $(\mathrm{RR}$, 1.11; $95 \%$ CI, 0.90 to $1.37 ; \mathrm{I}^{2}=39 \%$ ) (Fig. 2). 


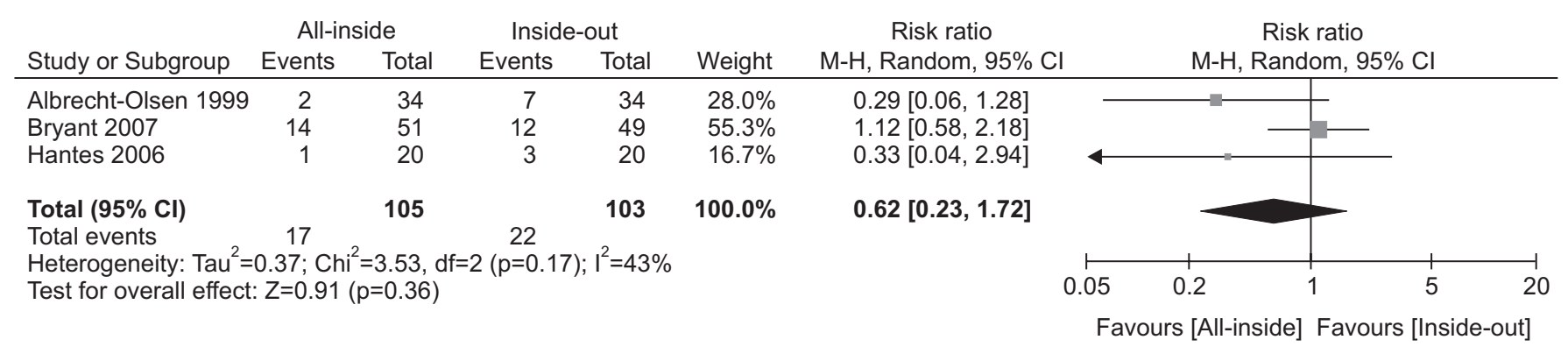

Fig. 3. Forest plot of the prevalence of perioperative complications in the randomized controlled trials using the all-inside and inside-out techniques. CI: confidence interval.

\section{2) Perioperative complications}

In the qualitative analysis, regardless of suture technique, the prevalence of perioperative complications was $2.2 \%-26.0 \%$ across studies. The three $\mathrm{RCTs}^{20,21,25)}$ reported on the perioperative complications between the two techniques with a total of 208 patients (105 in the all-inside group and 103 in the inside-out group). There were no significant differences in the perioperative complications between the all-inside and inside-out techniques (RR, 0.62; 95\% CI, 0.23 to $1.72 ; \mathrm{I}^{2}=43 \%$ ) (Fig. 3 ).

\section{Discussion}

In this meta-analysis, we assessed evidence from clinical studies that evaluated the outcomes of arthroscopic meniscus repair for meniscus tears in young patients, and we compared the effects between the all-inside and inside-out meniscal suture techniques. The most important finding of the present study was that the clinical outcomes such as meniscus healing rates and perioperative complications in these patients improved favorably after arthroscopic meniscus repair, and there were no significant differences between the two different suture technique groups. This indicates that arthroscopic meniscus repair should be considered a treatment of choice for traumatic meniscus tears in young patients and the suture technique can be selected based on the surgeon's familiarity with the procedure.

In terms of anatomic characteristics of the meniscus, the peripheral one third of the meniscus has the best blood supply in adults $^{28}$. For this reason, vertical tears $<5 \mathrm{~mm}$ in length in the peripheral one third of the meniscus are generally stable, and conservative care is recommended ${ }^{6}$. However, whereas conservative treatment of stable meniscus tears can potentially result in complete healing, sometimes it leads to a reparable or irreparable tear if the tear progressed due to highly demanding activity or frequent trauma history, especially in young patients; in these cases, arthroscopic meniscus repair is recommended for conserv- ing the remaining meniscus tissue. Meniscus repair preserves meniscus tissue, and it also offers biomechanical advantages ${ }^{7)}$. In the same context, multiple studies have presented satisfactory outcomes after meniscus suture repair: in one meta-analysis, meniscus repair was associated with better long-term outcomes than those following meniscectomy ${ }^{10)}$. Thus, despite the results of previous studies showing a failure rate of $15 \%-30 \%$ in repaired isolated meniscal tears without ACL injury, many authors advocate meniscus repair to preserve meniscus tissue ${ }^{29,30}$. Our results also showed favorable healing rates and perioperative complication rates after arthroscopic meniscus repair in young patients. This can strengthen the clinical evidence for arthroscopic meniscus repair in these patients.

Meniscus repair is globally accepted among orthopedic surgeons, and the inside-out technique has been used widely for posterior horn tears of the meniscus; however, this technique requires an additional skin incision and has a risk of neurovascular injuries and postoperative stiffness ${ }^{27}$. The new all-inside technique using bioabsorbable materials was developed to overcome the drawbacks ${ }^{19)}$. The all-inside technique also has benefits such as short operation time and good healing rate with satisfactory outcomes ${ }^{31}$. Furthermore, this technique was especially useful in cases with ramp lesions ${ }^{277}$. Despite these various benefits of the all-inside technique, several complications are associated with this technique as well. For example, authors of one study reported complications such as chondral damage, device-induced irritation, device breakage, foreign body reaction, and synovitis ${ }^{32-35}$. Based on these discrepancies, one study ${ }^{36}$ demonstrated that the all-inside technique using arrows had significantly higher failure strength than did other meniscal repair devices; conversely, another study ${ }^{37)}$ found that inside-out sutures had significantly higher mean loads to failure than meniscal arrows. In addition, Spindler et al. ${ }^{24)}$ found no differences in failure rates between two groups, and Bryant et al. ${ }^{20)}$ conducted RCTs on this topic and also reported that there were no statistically significant differences in 
measured outcomes between the meniscus inside-out suturing and arrows. In the same context, Spindler et al. ${ }^{24)}$ and Bryant et $\mathrm{al}^{20)}$ reported that there were no differences in meniscus healing rates or perioperative complications between the two groups in their meta-analyses. Although we could not analyze clinical outcome scales in this meta-analysis due to insufficient data, our results strengthen previous study results and confirm the appropriateness of arthroscopic meniscus suture repair for treating meniscus tears in young patients. To verify which suture technique yields greater improvement in clinical outcomes, more high quality RCTs are needed.

We assessed the quality of the included studies using the PEDro critical appraisal scoring system or the Newcastle-Ottawa Quality Assessment Scale. By PEDro score, all RCTs scored $\geq 8$ points (range, 8 to 9 points) and Newcastle-Ottawa Quality Assessment Scale for comparative studies scored $\geq 7$ points (range, 7 to 8 points). These results indicate a low risk of bias of the included studies and their eligibility for the analysis. In addition, two independent, blinded reviewers performed the screening and data extraction, which is one of the strengths of our study.

However, despite its strengths, there are some limitations to the present study. First, we used a relatively small number of studies in this meta-analysis: a search of the literature revealed that the number of original, previously published articles on this topic is inadequate, which is a clear limitation. However, all of the included clinical studies were entirely level I or II (high-quality) studies, and this may carry a low risk of bias. Second, there was heterogeneity in the studies regarding the patient populations, the different scoring systems, the patterns of meniscus tears, the follow-up durations, and the surgical indications for arthroscopic meniscus surgery. Third, we did not fully consider other factors that could have affected clinical outcomes, such as patient gender, meniscus tear type, size, location (medial or lateral) and site, time from injury to arthroscopic surgery, and concomitant ACL injury. Of course, removing all confounding factors is ideal to reduce the risk of bias in the evaluation of one independent factor. However, strict control of all confounding factors affecting clinical outcomes is difficult in practice. Furthermore, permission to conduct more dissimilar heterogeneous studies will lead to improved external validity and generalizability. This concept is associated with "effectiveness": heterogeneous, more practical, "real world" studies in normal clinical conditions likely encountered in practical clinical trials ${ }^{38)}$. Hence, even though all of the included studies are prospective studies, the findings of the forest plots of the present study should be interpreted with great caution considering that the data were extracted from somewhat heterogeneous stud- ies. Furthermore, despite these discrepancies, to minimize the risk of bias and compensate for heterogeneity, we used random effect model analysis according to the Cochrane Guidelines. In the future, in order to overcome these issues, prospective studies that control for these independent factors through high-quality medical research need to be encouraged.

\section{Conclusions}

The present study presents favorable clinical outcomes in terms of meniscus healing rates and perioperative complications in young patients. Furthermore, based on our results, both the allinside and inside-out meniscal suture techniques can be equally effective in these patients. To strengthen our results, more highquality clinical trials and RCTs are warranted.

\section{Conflict of Interest}

No potential conflict of interest relevant to this article was reported.

\section{References}

1. Hollis JM, Pearsall AW 4th, Niciforos PG. Change in meniscal strain with anterior cruciate ligament injury and after reconstruction. Am J Sports Med. 2000;28:700-4.

2. Renstrom P, Johnson RJ. Anatomy and biomechanics of the menisci. Clin Sports Med. 1990;9:523-38.

3. Seedhom BB, Dowson D, Wright V. Proceedings: functions of the menisci: a preliminary study. Ann Rheum Dis. 1974; 33:111.

4. Voloshin AS, Wosk J. Shock absorption of meniscectomized and painful knees: a comparative in vivo study. J Biomed Eng. 1983;5:157-61.

5. Zimny ML, Albright DJ, Dabezies E. Mechanoreceptors in the human medial meniscus. Acta Anat (Basel). 1988;133: $35-40$.

6. Giuliani JR, Burns TC, Svoboda SJ, Cameron KL, Owens BD. Treatment of meniscal injuries in young athletes. J Knee Surg. 2011;24:93-100.

7. Baratz ME, Fu FH, Mengato R. Meniscal tears: the effect of meniscectomy and of repair on intraarticular contact areas and stress in the human knee: a preliminary report. Am J Sports Med. 1986;14:270-5.

8. Renstrom P, Ljungqvist A, Arendt E, Beynnon B, Fukubayashi T, Garrett W, Georgoulis T, Hewett TE, Johnson 
R, Krosshaug T, Mandelbaum B, Micheli L, Myklebust G, Roos E, Roos H, Schamasch P, Shultz S, Werner S, Wojtys E, Engebretsen L. Non-contact ACL injuries in female athletes: an International Olympic Committee current concepts statement. Br J Sports Med. 2008;42:394-412.

9. Lee SJ, Aadalen KJ, Malaviya P, Lorenz EP, Hayden JK, Farr J, Kang RW, Cole BJ. Tibiofemoral contact mechanics after serial medial meniscectomies in the human cadaveric knee. Am J Sports Med. 2006;34:1334-44.

10. Xu C, Zhao J. A meta-analysis comparing meniscal repair with meniscectomy in the treatment of meniscal tears: the more meniscus, the better outcome? Knee Surg Sports Traumatol Arthrosc. 2015;23:164-70.

11. Haas AL, Schepsis AA, Hornstein J, Edgar CM. Meniscal repair using the FasT-Fix all-inside meniscal repair device. Arthroscopy. 2005;21:167-75.

12. Barber FA, Herbert MA. Meniscal repair devices. Arthroscopy. 2000;16:613-8.

13. Albrecht-Olsen PM, Bak K. Arthroscopic repair of the bucket-handle meniscus: 10 failures in 27 stable knees followed for 3 years. Acta Orthop Scand. 1993;64:446-8.

14. Farng E, Sherman O. Meniscal repair devices: a clinical and biomechanical literature review. Arthroscopy. 2004;20:27386.

15. Tsai AM, McAllister DR, Chow S, Young CR, Hame SL. Results of meniscal repair using a bioabsorbable screw. Arthroscopy. 2004;20:586-90.

16. Fairbank TJ. Knee joint changes after meniscectomy. J Bone Joint Surg Br. 1948;30:664-70.

17. Jackson JP. Degenerative changes in the knee after meniscectomy. Br Med J. 1968;2:525-7.

18. Jorgensen U, Sonne-Holm S, Lauridsen F, Rosenklint A. Long-term follow-up of meniscectomy in athletes: a prospective longitudinal study. J Bone Joint Surg Br. 1987;69:803.

19. Jurist KA, Greene PW 3rd, Shirkhoda A. Peroneal nerve dysfunction as a complication of lateral meniscus repair: a case report and anatomic dissection. Arthroscopy. 1989;5:141-7.

20. Bryant D, Dill J, Litchfield R, Amendola A, Giffin R, Fowler P, Kirkley A. Effectiveness of bioabsorbable arrows compared with inside-out suturing for vertical, reparable meniscal lesions: a randomized clinical trial. Am J Sports Med. 2007;35: 889-96.

21. Hantes ME, Zachos VC, Varitimidis SE, Dailiana ZH, Karachalios T, Malizos KN. Arthroscopic meniscal repair: a comparative study between three different surgical techniques.
Knee Surg Sports Traumatol Arthrosc. 2006;14:1232-7.

22. Maher CG, Sherrington C, Herbert RD, Moseley AM, Elkins M. Reliability of the PEDro scale for rating quality of randomized controlled trials. Phys Ther. 2003;83:713-21.

23. Stang A. Critical evaluation of the Newcastle-Ottawa scale for the assessment of the quality of nonrandomized studies in meta-analyses. Eur J Epidemiol. 2010;25:603-5.

24. Spindler KP, McCarty EC, Warren TA, Devin C, Connor JT. Prospective comparison of arthroscopic medial meniscal repair technique: inside-out suture versus entirely arthroscopic arrows. Am J Sports Med. 2003;31:929-34.

25. Albrecht-Olsen P, Kristensen G, Burgaard P, Joergensen $\mathrm{U}$, Toerholm C. The arrow versus horizontal suture in arthroscopic meniscus repair: a prospective randomized study with arthroscopic evaluation. Knee Surg Sports Traumatol Arthrosc. 1999;7:268-73.

26. Barber FA, Johnson DH, Halbrecht JL. Arthroscopic meniscal repair using the BioStinger. Arthroscopy. 2005;21:744-50.

27. Choi NH, Kim TH, Victoroff BN. Comparison of arthroscopic medial meniscal suture repair techniques: insideout versus all-inside repair. Am J Sports Med. 2009;37:214450 .

28. Arnoczky SP, Warren RF. Microvasculature of the human meniscus. Am J Sports Med. 1982;10:90-5.

29. Rockborn P, Messner K. Long-term results of meniscus repair and meniscectomy: a 13-year functional and radiographic follow-up study. Knee Surg Sports Traumatol Arthrosc. 2000;8:2-10.

30. Stein T, Mehling AP, Welsch F, von Eisenhart-Rothe R, Jäger A. Long-term outcome after arthroscopic meniscal repair versus arthroscopic partial meniscectomy for traumatic meniscal tears. Am J Sports Med. 2010;38:1542-8.

31. Hurel C, Mertens F, Verdonk R. Biofix resorbable meniscus arrow for meniscal ruptures: results of a 1-year follow-up. Knee Surg Sports Traumatol Arthrosc. 2000;8:46-52.

32. Anderson K, Marx RG, Hannafin J, Warren RF. Chondral injury following meniscal repair with a biodegradable implant. Arthroscopy. 2000;16:749-53.

33. Ganko A, Engebretsen L. Subcutaneous migration of meniscal arrows after failed meniscus repair. A report of two cases. Am J Sports Med. 2000;28:252-3.

34. Kurzweil PR, Tifford CD, Ignacio EM. Unsatisfactory clinical results of meniscal repair using the meniscus arrow. Arthroscopy. 2005;21:905.

35. Song EK, Lee KB, Yoon TR. Aseptic synovitis after meniscal repair using the biodegradable meniscus arrow. Arthros- 
copy. 2001;17:77-80.

36. Arnoczky SP, Lavagnino M. Tensile fixation strengths of absorbable meniscal repair devices as a function of hydrolysis time: an in vitro experimental study. Am J Sports Med. 2001; 29:118-23.

37. Dervin GF, Downing KJ, Keene GC, McBride DG. Failure strengths of suture versus biodegradable arrow for meniscal repair: an in vitro study. Arthroscopy. 1997;13:296-300.

38. Harris JD, Brand JC, Cote MP, Dhawan A. Research pearls: the significance of statistics and perils of pooling. Part 3: pearls and pitfalls of meta-analyses and systematic reviews. Arthroscopy. 2017;33:1594-602. 


\section{Kang et al. All-Inside vs. Inside-Out Suture in Arthroscopic Meniscus Repair}

Appendix 1. Electronic Search Strategy for Each Database

\section{MEDLINE}

1. "Menisci, Tibial"[Mesh] 6150

2. Menisci[tiab] OR meniscal[tiab] OR meniscus[tiab] 12783

3. 1 OR 214102

4. ((“Arthroscopy”[Mesh]) OR "Suture Techniques"[Mesh]) OR "Rupture"[Majr] 76936

5. repairs[tiab] OR tears[tiab] OR Arthroscopy[tiab] OR Arthroscopies[tiab] OR Arthroscopic[tiab] OR Rupture[tiab] OR Suture[tiab] OR repair[tiab] OR tear[tiab] OR Rupture[tiab] OR injury[tiab] OR injuries[tiab] 1016833

6. 4 OR 51047348

7. 3 AND 68475

8. 7 NOT ("review"[Publication Type] OR "review literature as topic"[MeSH Terms]) 7463

9. 8 AND (“Controlled clinical trial” [ptyp]) 275

\section{EMBASE}

1. 'knee meniscus'/exp 12026

2. Menisci:ab,ti OR meniscal:ab,ti OR meniscus:ab,ti 15538

3. 1 OR 218213

4. 'knee meniscus rupture'/exp OR 'knee arthroscopy'/exp OR 'suturing method'/exp OR 'suture'/de OR 'meniscal repair'/exp OR 'meniscal surgery'/de 68017

5. repairs:ab,ti OR tears:ab,ti OR Arthroscopy:ab,ti OR Arthroscopies:ab,ti OR Arthroscopic:ab,ti OR Rupture:ab,ti OR Suture:ab,ti OR repair:ab,ti OR tear:ab,ti OR Rupture:ab,ti OR injury:ab,ti OR injuries:ab,ti 1260152

6. 4 OR 51288683

7. 3 AND 611581

8. 7 NOT ('conference review'/it OR 'review'/it) 10300

9. 8 AND ('controlled study'/it) 2377

\section{COCHRANE}

1. MeSH descriptor: [Menisci, Tibial] explode all trees 219

2. Menisci OR meniscal OR meniscus 702

3. 1 OR 2702

4. MeSH descriptor: [Arthroscopy] explode all trees 1443

5. MeSH descriptor: [Suture Techniques] explode all trees 1976

6. MeSH descriptor: [Rupture] this term only 615

7. repairs OR tears OR Arthroscopy OR Arthroscopies OR Arthroscopic OR Rupture OR Suture OR repair OR tear OR Rupture OR injury OR injuries 52836

8. 4-7/OR 53028

9. 3 AND 8587

10. 9/TRIALS 549 


\section{Web of Science (WOS)}

1. TOPIC: (Menisci OR meniscal OR meniscus) OR TITLE: (Menisci OR meniscal OR meniscus) 15299

2. TOPIC: (repairs OR tears OR Arthroscopy OR Arthroscopies OR Arthroscopic OR Rupture OR Suture OR repair OR tear OR Rupture OR injury OR injuries) OR TITLE: (repairs OR tears OR Arthroscopy OR Arthroscopies OR Arthroscopic OR Rupture OR Suture OR repair OR tear OR Rupture OR injury OR injuries) 1218242

3. 1 AND 27014

4. 3 Refined by: [excluding] DOCUMENT TYPES: (REVIEW) 6576

\section{SCOPUS}

1. INDEXTERMS("Menisci, Tibial") 6972

2. TITLE-ABS(Menisci OR meniscal OR meniscus) 22154

3. 1 OR 223706

4. INDEXTERMS(“Arthroscopy” OR “Suture Techniques" OR "Rupture”) 184410

5. TITLE-ABS(repairs OR tears OR Arthroscopy OR Arthroscopies OR Arthroscopic OR Rupture OR Suture OR repair OR tear OR Rupture OR injury OR injuries) 1383260

6. 4 OR 51447468

7. 3 AND 611082

8. 7 AND ( EXCLUDE (DOCTYPE, "re”) ) 9937

9. 8 AND (LIMIT-TO (DOCTYPE, “ar”)) 8869 